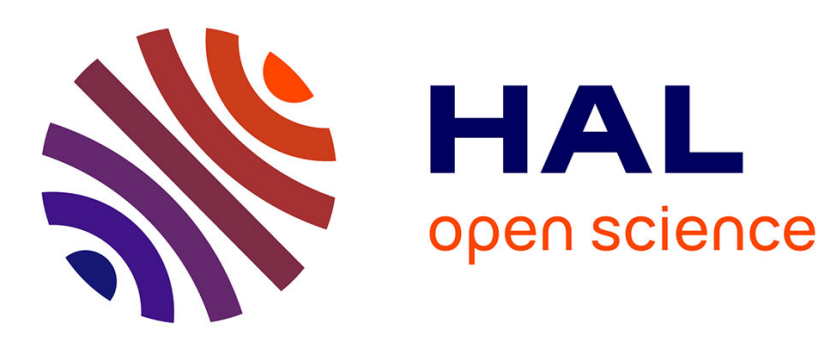

\title{
Commande optimale d'un système générateur photovoltaïque-convertisseur statique - récepteur
}

M. Barlaud, B. de Fornel, M. Gauvrit, J.P. Requier

\section{To cite this version:}

M. Barlaud, B. de Fornel, M. Gauvrit, J.P. Requier. Commande optimale d'un système générateur photovoltaïque-convertisseur statique - récepteur. Revue de Physique Appliquée, 1984, 19 (10), pp.905915. 10.1051/rphysap:019840019010090500 . jpa-00245282

\section{HAL Id: jpa-00245282 https://hal.science/jpa-00245282}

Submitted on 1 Jan 1984

HAL is a multi-disciplinary open access archive for the deposit and dissemination of scientific research documents, whether they are published or not. The documents may come from teaching and research institutions in France or abroad, or from public or private research centers.
L'archive ouverte pluridisciplinaire HAL, est destinée au dépôt et à la diffusion de documents scientifiques de niveau recherche, publiés ou non, émanant des établissements d'enseignement et de recherche français ou étrangers, des laboratoires publics ou privés. 
Classification

Physics Abstracts

$83.40-83.60$

\title{
Commande optimale d'un système générateur photovoltaïque - convertisseur statique - récepteur
}

\author{
M. Barlaud ( $\left.{ }^{1}\right)$, B. de Fornel $\left({ }^{2}\right)$, M. Gauvrit $\left({ }^{3}\right)$ et J. P. Requier $\left({ }^{4}\right)$ \\ ( $\left.{ }^{1}\right)$ Agence Française pour la Maîtrise de l'Energie, Solaire et Géothermie, \\ Route des Lucioles, 06565 Valbonne, France \\ $\left({ }^{2}\right)$ ENSEEIHT, Laboratoire d'Electrotechnique et d'Electronique Industrielle ( ${ }^{*}$ ), \\ 2, rue Camichel, 31071 Toulouse Cedex, France \\ $\left({ }^{3}\right)$ CERT-DERA, 2, avenue Edouard Belin, BP 4025, 31055 Toulouse Cedex, France \\ $\left({ }^{4}\right)$ Université Paris-Val de Marne, avenue du Général de Gaulle, 94000 Créteil, France
}

(Reçu le 3 mai 1983, révisé le 19 mars 1984 et le 13 juin 1984, accepté le 15 juin 1984)

\begin{abstract}
Résumé. - En régime permanent, un convertisseur statique peut assurer le transfert optimal de puissance entre une source de puissance finie et un récepteur.

L'étude de ce transfert en régime dynamique fait apparaître une interaction entre le récepteur et le générateur qui provoque des réponses non minimales de phase dues à la présence de zéros à partie réelle positive dans la fonction de transfert du système.

Afin d'optimiser le transfert dynamique de puissance, les auteurs proposent des critères de choix des paramètres de structure, du nombre de degrés de liberté et des lois de commande de convertisseurs qui garantissent des réponses minimales de phase.

Ensuite, une compensation par retour d'état permet d'assurer un transfert optimal de la puissance entre le générateur et le récepteur.
\end{abstract}

\begin{abstract}
In a steady state, a static converter can assure the optimal transfer of power between a finite source of power and a receiver.

The study of this transfer in a dynamic state presents an interaction between the receiver and generator which provokes non-minimal phasal responses due to the presence of « zeros » with the positive real set in the transfer function of the system.

So as to optimize the dynamic transfer of power, the authors propose chosen criteria for structural parameters, in numbers of « degrees of liberty » and command laws for converters which guarantee minimal phase responses.
\end{abstract}

\section{Introduction.}

Pour résoudre le problème du transfert de la puissance optimale d'un générateur électrique vers un récepteur électromécanique, il est généralement nécessaire d'intercaler un convertisseur statique commandé par des algorithmes de recherche extrémale adaptative [1-3].

Ces algorithmes étant fondés sur des méthodes de perturbations, l'étude des réponses transitoires de ces systèmes revêt une importance particulière tant pour l'optimisation du transfert dynamique de puissance que pour leurs bonnes stabilité et fiabilité de fonctionnement.

Le premier but de cet article est d'étudier les zéros de la matrice de transfert d'ensembles générateur

\section{(*) ERA-CNRS No 536.}

photovoltaïque-convertisseur-récepteur afin d'éviter des réponses transitoires non minimales de phase qui engendrent des perturbations. En effet, l'inversion momentanée de certaines variables d'état peut nuire au bon fonctionnement du système.

Le premier exemple traité (Fig. 1) est celui d'un ensemble : générateur photovoltaïque-hacheur à transistor-charge résistive. Le convertisseur n'ayant qu'un seul degré de liberté, on démontre alors que les réponses dynamiques sont non minimales de phase.

Afin de palier à ce phénomène, on propose d'utiliser un convertisseur statique à deux degrés de liberté. L'ensemble étudié est composé :

- d'un générateur photovoltaïque,

- de deux hacheurs montés en série,

- d'une charge résistive. 


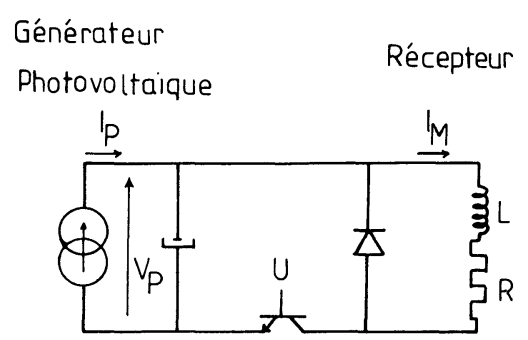

Fig. 1. - Synoptique du montage - Générateur-hacheur -charge résistive.

[Photovoltaic cell array - Chopper resistive load.]

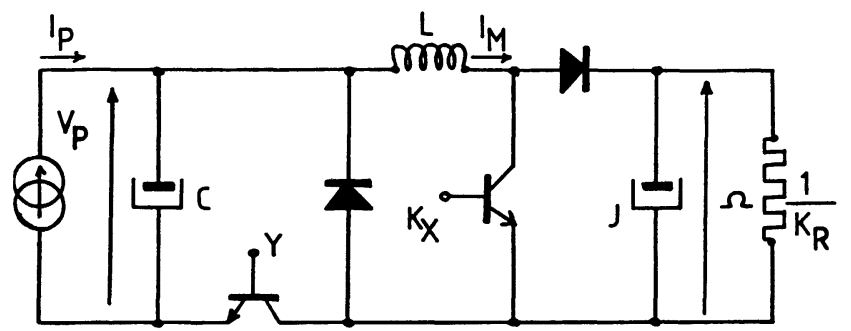

Photopiles

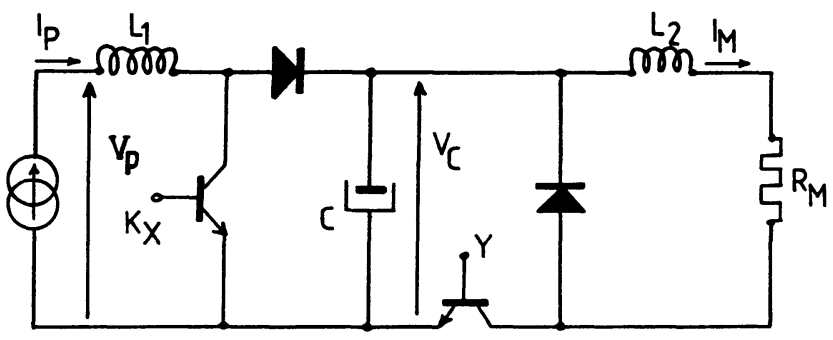

Photopiles

Fig. 2. - Générateur photovoltaïque avec un système à double commande.

[Photovoltaic generator with a double control system.]

On démontre alors qu'il est possible de trouver des commandes simultanées, définies par un plan de commande, qui garantissent des réponses minimales de phase.

Il est possible d'établir une équivalence formelle entre ce dernier montage et l'ensemble générateur photovoltaïque-hacheur-machine continue à excitation séparée, représenté figure 3. Le deuxième degré de liberté provient de l'action sur le flux magnétique de la machine.

La mise en équation sous forme de représentation d'état permet d'obtenir les matrices de transfert de ces ensembles en vue d'en étudier les zéros. Ensuite, le deuxième but poursuivi dans cette étude est de déterminer par les techniques d'état, une loi de commande optimale du système. Pour cela, on utilise la technique de compensation par retour d'état.

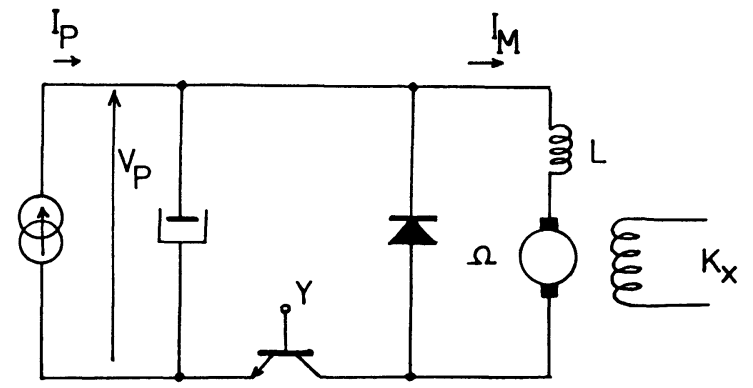

Photopiles

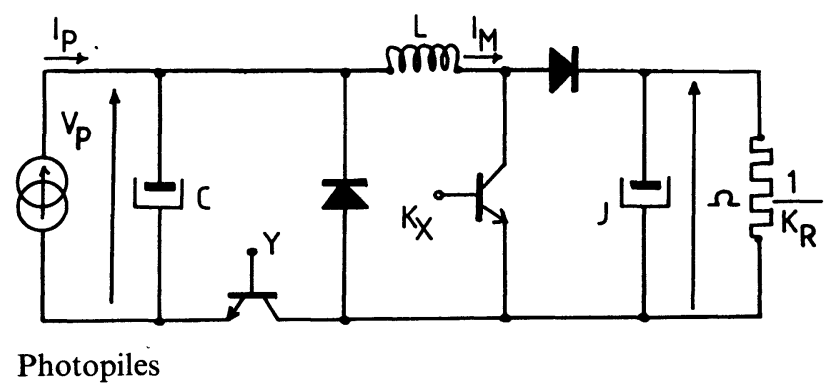

Fig. 3. - Générateur photovoltaïque hacheur-moteur à courant continu.

[Photovoltaic generator-Chopper-Dc motor.]

\section{Etude de la structure : générateur photovoltaïque- hacheur-charge résistive.}

A l'aide du montage représenté figure 1 , nous allons montrer qu'il n'est pas possible d'obtenir des réponses minimales de phase en intercalant entre un générateur et une charge, un convertisseur ayant un seul degré de liberté.

2.1 Mise EN ÉQUATION. - Le générateur photovoltaïque est décrit par sa caractéristique statique non linéaire $I_{\mathrm{p}}=f\left(V_{\mathrm{p}}\right)$.

Son équation de fonctionnement est :

$$
I_{\mathrm{p}}=I_{0}-I_{\mathrm{s}}\left(\exp \left(q \cdot V_{\mathrm{p}} / k T\right)-1\right)
$$

où $I_{0}$ est le photocourant proportionnel à l'éclairement, $I_{\mathrm{p}}$ le courant pour une tension de fonctionnement $V_{\mathrm{p}}$.

Pour un point de fonctionnement statique $\mathbf{M}_{0}$ défini par la tension $V_{\mathrm{p}_{0}}$ et le courant $I_{\mathrm{p}_{0}}$, soit : $R_{\mathrm{s}}=\frac{V_{\mathrm{p}_{0}}}{I_{\mathrm{p}_{\mathrm{o}}}}$ sa résistance statique et $R_{\mathrm{g}}=\left(-\frac{\partial V_{\mathrm{p}}}{\partial I_{\mathrm{p}}}\right)_{\mathrm{M}_{\mathrm{o}}}$ sa résistance dynamique, notons que lorsque ce générateur fonctionne à son optimum de puissance, sa résistance statique $R_{\mathrm{s}}$ est égale à sa résistance dynamique $\boldsymbol{R}_{\mathbf{g}}$

- Nous nous plaçons dans des conditions de fonctionnement en conduction continue, permettant de considérer le hacheur comme un système linéaire. (Cf. Middle-Brook and Slobodan Cuk [4].) 
De plus, négligeant les pertes, on a un fonctionnement statique, au point $M_{0}$ du générateur :

$$
\begin{gathered}
V_{\mathrm{M}_{0}}=U_{0} \cdot V_{\mathrm{p}_{0}} \\
I_{\mathrm{p}_{0}}=U_{0} \cdot I_{\mathrm{M}_{0}}
\end{gathered}
$$

où $U_{0}$ est le rapport cyclique de conduction.

Ces relations conduisent à l'équation de fonctionnement :

$$
U_{0}^{2}=R_{\mathrm{M}} / R_{\mathrm{s}}
$$

Nous avons le système d'équations :

$$
\stackrel{\circ}{X}=A x+B U \quad Z=C X
$$

avec :

$$
A=a(i, j)=\left(\frac{\partial f_{i}}{\partial x_{j}}\right)_{\mathrm{M}_{0}}\left|\begin{array}{cc}
-\frac{1}{R_{\mathrm{g}} C} & -\frac{U_{0}}{C} \\
\frac{U_{0}}{L} & -\frac{R_{\mathrm{M}}}{L}
\end{array}\right| \quad B=b(i, j)=\left(\frac{\partial f_{i}}{\partial u_{j}}\right)\left|\begin{array}{c}
-\frac{I_{\mathrm{M}_{0}}}{C} \\
\frac{V_{\mathrm{p}_{0}}}{L}
\end{array}\right|
$$

La matrice de sortie $C$ est la matrice identité.

2.2 ETUde DE LA STABILITÉ. - L'étude de la stabilité est un préalable à toute investigation ultérieure.

La détermination des racines du polynôme caractéristique $P(\lambda)$ permet d'étudier la stabilité du système :

$$
P(\lambda)=\operatorname{Det}(\lambda I-A)=\lambda^{2}+a_{1} \lambda+a_{0}=0
$$

où :

$$
a_{1}=\left(\frac{R_{\mathrm{M}}}{L}+\frac{1}{C R_{\mathrm{g}}}\right) \text { et } a_{0}=\frac{1}{L C}\left(U_{0}^{2}+\frac{R_{\mathrm{M}}}{R_{\mathrm{g}}}\right) \text {. }
$$

Pour un générateur photovoltaïque, la résistance $R_{\mathrm{g}}$ est toujours positive, le système est toujours stable.

2.3 ETUDE DES ZÉROS DE LA FONCTION DE TRANSFERT. - Dans ce cas simple, la fonction de transfert du système peut être établie directement par substitution :

$$
T(p)=\frac{I_{\mathrm{M}}(p)}{u(p)}\left[\frac{V_{\mathrm{p}_{0}}}{L} \cdot p+\frac{V_{\mathrm{p}_{0}}}{L C}\left(\frac{1}{R_{\mathrm{g}}}-\frac{1}{R_{\mathrm{s}}}\right)\right] \frac{1}{\psi(p)}
$$

où :

$$
\psi(p)=\operatorname{Det}(p . I-A) .
$$

Le zéro de cette fonction est alors :

$$
Z_{1}=\frac{1}{C}\left(\frac{1}{R_{\mathrm{s}}}-\frac{1}{R_{\mathrm{g}}}\right)
$$

- Les équations côté générateur et côté récepteur sont donc :

$$
\begin{aligned}
& \frac{\mathrm{d} V_{\mathrm{p}}}{\mathrm{d} t}=\frac{I_{\mathrm{p}}}{C}-\frac{I_{\mathrm{M}}}{C} \cdot U_{0} \\
& \frac{\mathrm{d} I_{\mathrm{M}}}{\mathrm{d} t}=\frac{V_{\mathrm{p}}}{L} \cdot U_{0}-\frac{R_{\mathrm{M}}}{L} \cdot I_{\mathrm{M}} .
\end{aligned}
$$

- La linéarisation de ce système d'équation $\stackrel{\circ}{X}=f(X, V)$ autour du point de fonctionnement statique $\mathbf{M}_{0}$ est obtenue en calculant son jacobien.

\section{Par conséquent :}

- La réponse est minimale de phase si :

$$
R_{\mathrm{g}}<R_{\mathrm{s}} \Leftrightarrow V_{\mathrm{p}}>V_{\text {popt }} .
$$

- La réponse est non minimale de phase et il y a inversion de la variable d'état $I_{M}(p)$ si :

$$
R_{\mathrm{g}}>R_{\mathrm{s}} \Leftrightarrow V_{\mathrm{p}}<V_{\text {popt }} .
$$

La figure 4 représente des courbes de réponse non minimales de phase obtenues par intégration numérique de l'équation d'état.

2.4 ConClusion. - Le transfert optimal de puissance entre un générateur photovoltaïque et une charge résistive ne peut être réalisé à l'aide d'un convertisseur n'ayant qu'un seul degré de liberté (convertisseur monocommande) car il entraîne une réponse non minimale de phase de la variable d'état du récepteur $I_{\mathrm{M}}(t)$.

Afin d'éviter toute réponse non minimale de phase, nous proposons des structures et des lois de commande de convertisseurs à plusieurs degrés de liberté (convertisseurs multicommandes).

3. Etude d'une structure de convertisseur et de ses lois de commande assurant des réponses minimales de phase.

On montre qu'en choisissant convenablement les 
notations, il existe une équivalence formelle entre les montages de la figure 3. La machine à courant continu à excitation séparée est équivalente à un hacheur survolteur.

Nous avons choisi d'effectuer la mise en équation sur le montage hacheur dévolteur machine à courant continu.

3. 1 MiSE en ÉQUATION DE L'ENSEMBLE DE LA FIGURE 3. a) Equations en régime permanent. - Soit $E_{0}$ la f.é.m. de la machine à courant continu, $C_{\mathrm{R}}$ le couple mécanique résistant, $\Omega_{0}$ sa vitesse de rotation et $I_{\mathrm{M}_{0}}$ le courant d'induit. Les équations de fonctionnement sont alors :

$$
E_{0}=K_{\mathrm{x}} \Omega_{0}
$$

On choisit un couple résistant proportionnel à la vitesse de rotation :

$$
C_{\mathrm{R}}=K_{\mathrm{s}} \Omega_{0}
$$

En négligeant les pertes mécaniques et magnétiques de la machine, le couple électromagnétique en régime permanent a pour expression :

$$
C_{\mathrm{em}}=K_{\mathrm{x}} I_{\mathrm{M}_{\mathrm{o}}}=K_{\mathrm{s}} \Omega_{0} .
$$

En régime permanent, on en déduit l'équation de fonctionnement :

$$
U_{0}=\left(R_{\mathrm{M}}+\frac{K_{\mathrm{x}}^{2}}{K_{\mathrm{s}}}\right) / R_{\mathrm{s}_{0}}
$$

où $R_{\mathrm{s}_{0}}$ est la résistance statique au point de fonctionnement.

b) Régime transitoire. - Nous supposerons la fréquence de hachage suffisamment grande pour pouvoir considérer le hacheur comme un amplificateur de gain $Y$. Les équations de fonctionnement sont donc :

$$
\begin{aligned}
\frac{\mathrm{d} V_{\mathrm{p}}}{\mathrm{d} t} & =\frac{I_{\mathrm{p}}}{c}-\frac{U_{0}}{C} I_{\mathrm{M}} \\
\frac{\mathrm{d} I_{\mathrm{M}}}{\mathrm{d} t} & =\frac{U_{0} V_{\mathrm{p}}}{L}-\frac{R_{\mathrm{M}}}{L} I_{\mathrm{M}}-\frac{K_{\mathrm{x}}}{L} \Omega \\
\frac{\mathrm{d} \Omega}{\mathrm{d} t} & =\frac{K_{\mathrm{x}}}{J} I_{\mathrm{M}}-\frac{K_{\mathrm{R}}}{J} \Omega .
\end{aligned}
$$

Ce système $X=f(X, Y)$ est non linéaire. En linéarisant autour d'un point de fonctionnement défini par $V_{\mathrm{p}_{0}}, I_{\mathrm{M}_{0}}, \Omega_{0}, U_{0}$ on obtient le modèle d'état en calculant :

$$
\begin{aligned}
& \dot{X}=A X+B U \\
& Y=C X
\end{aligned}
$$

où

$A=a(i, j)=\left(\frac{\partial f_{i}}{\partial x_{j}}\right)=\left|\begin{array}{ccc}\frac{1}{R_{\mathrm{g}} C} & -\frac{U_{0}}{C} & 0 \\ \frac{U_{0}}{L} & -\frac{R_{\mathrm{M}}}{L} & -\frac{K_{\mathrm{x}}}{L} \\ 0 & \frac{K_{\mathrm{x}}}{J} & -\frac{K_{\mathrm{R}}}{J}\end{array}\right| C=I$.

La matrice $B$ dépend de la commande choisie. Il est possible de choisir ou bien d'agir simultanément sur :

- une commande $u_{1} \mathrm{du}$ rapport cyclique du hacheur dévolteur qui contrôle le courant d'induit de la machine, la matrice $B$ se réduit à $B_{1}$,

- une commande $u_{2}$ qui contrôle le flux de la machine continue ou le rapport cyclique du hacheur survolteur. La matrice $B_{2}$ définit cette commande.

$$
B_{1}=\left|\begin{array}{c}
-\frac{I_{\mathrm{M}_{0}}}{C} \\
\frac{V_{\mathrm{p}_{0}}}{L} \\
0
\end{array}\right| \quad B_{2}=\left|\begin{array}{c}
0 \\
-\frac{\Omega_{0}}{L} \\
\frac{I_{\mathrm{M}_{0}}}{J}
\end{array}\right| .
$$

3.2 Etude DE la STABiLité. - L'étude du signe des racines du polynôme caractéristique :

$$
\begin{aligned}
P(\lambda) & =\operatorname{Det}(\lambda I-A)= \\
& =\lambda^{3}+C_{2} \lambda^{2}+C_{1} \lambda+C_{0}=0
\end{aligned}
$$

avec :

$$
\begin{gathered}
C_{2}=\frac{R_{\mathrm{M}}}{L}+\frac{1}{C R_{\mathrm{g}}}+\frac{K_{\mathrm{R}}}{J} \\
C_{1}=\frac{R_{\mathrm{M}}}{R_{\mathrm{g}} L C}+\frac{K_{\mathrm{R}}}{J R_{\mathrm{g}} C}+\frac{R_{\mathrm{M}} K_{\mathrm{R}}}{L J}+\frac{K_{\mathrm{x}}^{2}}{L J}+\frac{U_{0}^{2}}{L C} \\
C_{0}=\frac{K_{\mathrm{x}}^{2}}{J L C R_{\mathrm{g}}}+\frac{K_{\mathrm{R}}}{J L C}\left(U_{0}^{2}+\frac{R_{\mathrm{M}}}{R_{\mathrm{g}}}\right)
\end{gathered}
$$

permet de conclure si $R_{\mathrm{g}}>0$ à une stabilité asymptotique du système.

Toutefois, la réponse du système à un échelon permet de distinguer deux modes de fonctionnement suivant la position du point de fonctionnement par rapport à la tension $V_{\text {popt }}$ :

- Le point de fonctionnement $V_{\mathrm{p}}$ est tel que $V_{\mathrm{p}}>V_{\mathrm{popt}}$ : la réponse est celle d'un système minimal de phase.

- Le point de fonctionnement $V_{\mathrm{p}}$ est tel que $V_{\mathrm{p}}<V_{\text {popt }}$ et alors la réponse à un échelon est celle 
d'un système non minimal de phase avec inversion de la réponse à l'origine (Fig. 4).

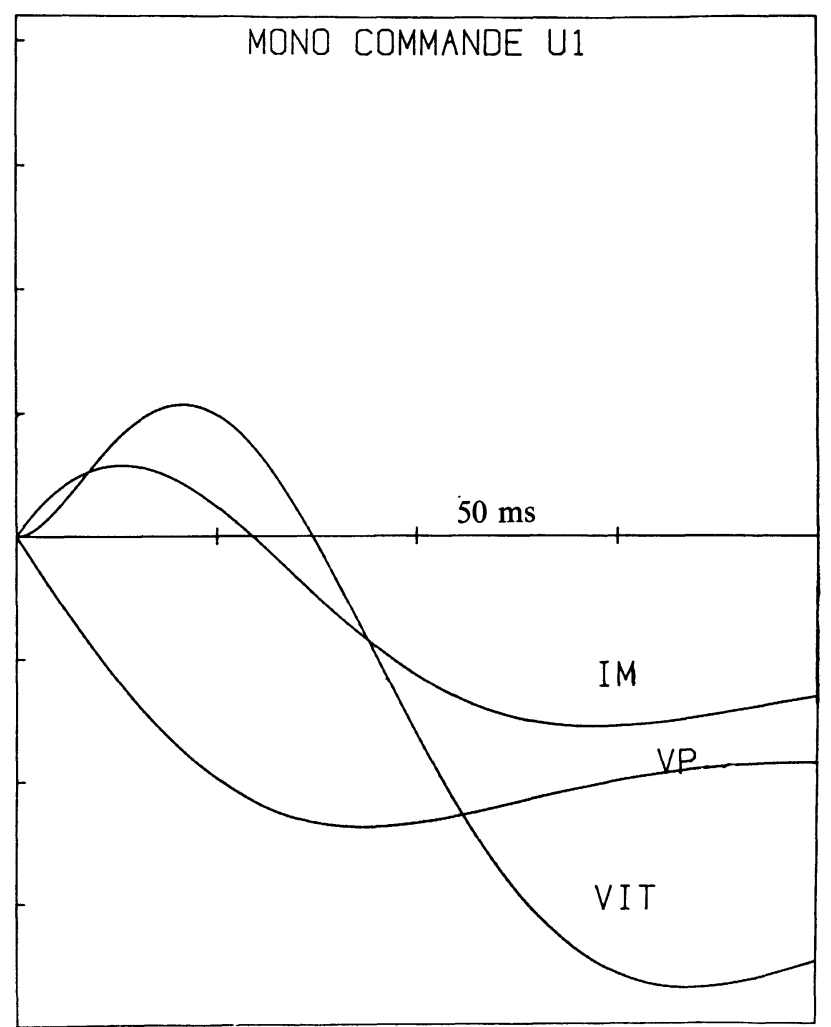

Fig. 4. - Réponse du système à un échelon $V_{\mathrm{po}}<V_{\mathrm{opt}}$. [Dynamic transient of the system for $V_{\mathrm{po}}<V_{\mathrm{opt}}$ ]

Ce phénomène est néfaste lorsqu'on effectue une recherche de puissance extrémale, car il provoque des perturbations du couple qui engendrent des vibrations mécaniques pouvant éventuellement entraîner la destruction de la machine. De plus, il empêche en régime dynamique, un transfert optimal de puissance entre le générateur et le récepteur. Ce phénomène est dû à la présence de zéros à parties réelles positives dans la fonction de transfert.

Une compensation par retour d'état ne modifiant pas les zéros, nous proposons une étude analytique des zéros et de l'influence des commandes et de la structure du montage.

3.3 Etude Des zéros. - Pour un système monovariable, les zéros sont les racines du numérateur de la fonction de transfert. En multivariables, la matrice de transfert est de dimension $(n \times m)(n$ dimension du système, $m$ nombre de commande). L'étude des zéros multivariables a fait l'objet de nombreuses recherches [5-7], malheureusement peu de résultats généraux ont été obtenus.

La définition du (ou des) zéro(s) en multivariable désormais généralement admise est la (ou les) valeur(s) des racines communes des numérateurs de la matrice de transfert. Les zéros, s'ils existent, sont invariants dans un retour d'état.

Toutefois, on peut toujours définir et étudier les zéros relatifs à une commande, l'autre restant nulle. Compte tenu des résultats obtenus dans chaque cas, l'introduction d'une relation entre les deux commandes $u_{1}$ et $u_{2}$ permet de se ramener au cas monovariable. Il serait extrêmement intéressant de pouvoir lier la nature des zéros obtenus avec chaque commande et la nature du zéro résultant des deux commandes liées. En particulier, si le système original est minimum de phase peut-on garantir aussi une réponse minimale de phase pour le système dont les entrées sont liées ? En fait, même dans ce cas le plus simple, il n'existe pas de théorie générale et Davison [7] a montré sur un contre-exemple que la proposition ci-dessus était fausse quelle que soit la relation entre les commandes et compte tenu d'un retour de sortie.

En l'absence de théorie générale, on se propose d'étudier les zéros du système pour chacune des commandes, ensuite une étude du zéro du système en fonction de la relation liant les deux commandes permettra de définir un plan de commande.

Nous proposons d'étudier les fonctions de transfert des trois grandeurs de sortie $\omega, i_{\mathrm{m}}$ et $V_{\mathrm{p}}$ successivement pour des commandes $u_{1}$ et $u_{2}$, en déduire l'influence des paramètres du montage. Lors d'une commande simultanée, nous définirons un plan de commande assurant un zéro stable.

La matrice de transfert s'obtient à partir de la représentation d'état :

$$
T(p)=C(p I-A)^{-1} B=\psi(p)^{-1} \cdot R(p) \cdot B
$$

avec

$$
\psi(p)=\operatorname{Det}(p I-A) \text { et } C \text { matrice des sorties . }
$$

Etude des zéros de la vitesse $\Omega$. - En fonction des deux commandes $u_{1}$ et $u_{2}$ la relation de transfert donnant la vitesse $\Omega$ est de la forme :

$$
\begin{aligned}
\Omega(p)=\left[\left(a_{0} p^{2}\right.\right. & \left.+a_{1} p+a^{2}\right) u_{1}(p)+ \\
& \left.+\left(b_{0} p^{2}+b_{1} p+b_{2}\right) u_{2}(p)\right] \cdot \psi(p)^{-1}
\end{aligned}
$$

avec

$$
\begin{aligned}
& a_{0}=0 \quad a_{1}=\frac{K_{\mathrm{x}} V_{\mathrm{p}_{0}}}{J L} \quad a_{2}=\frac{K_{\mathrm{x}} V_{\mathrm{p}_{0}}}{J L C}\left(\frac{1}{R_{\mathrm{g}}}-\frac{1}{R_{\mathrm{s}}}\right) \\
& b_{0}=\frac{I_{\mathrm{Mo}}}{J} \quad b_{1}=\frac{I_{\mathrm{M}_{0}}}{J}\left(\frac{1}{C R_{\mathrm{g}}}+\frac{R_{\mathrm{M}}}{L}-\frac{K_{\mathrm{x}}^{2}}{L K_{\mathrm{R}}}\right) \\
& b_{2}=\frac{I_{\mathrm{M}_{0}}}{J L C}\left(U_{0}^{2}+\frac{R_{\mathrm{M}}}{R_{\mathrm{g}}}-\frac{K_{\mathrm{x}}^{2}}{R_{\mathrm{g}} K_{\mathrm{R}}}\right) .
\end{aligned}
$$

Nous allons examiner les deux cas de fonctionnement avec un hacheur dévolteur et un hacheur survolteur. 
a) Commande par hacheur dévolteur.

La valeur du zéro est alors :

$$
z_{1}=\frac{1}{C}\left(\frac{1}{R_{\mathrm{s}}}-\frac{1}{R_{\mathrm{g}}}\right)
$$

La figure 5 représente la variation de la partie réelle du zéro en fonction de $V_{\mathrm{p}}$, point de fonctionnement. Il est à partie réelle négative, seulement si la tension de fonctionnement $V_{\mathrm{p}}$ est supérieure à la tension optimum.

\section{b) Commande par hacheur survolteur.}

Pour que les zéros soient à partie réelle négative, les contraintes inégalités suivantes doivent être vérifiées :

$$
\frac{1}{C R_{\mathrm{g}}}+\frac{R_{\mathrm{M}}}{L}-\frac{K_{\mathrm{x}}^{2}}{L K_{\mathrm{R}}} \geqslant 0 \Leftrightarrow \frac{L}{C}>R_{\mathrm{g}}\left(\frac{K_{\mathrm{x}}^{2}}{K_{\mathrm{R}}}-R_{\mathrm{M}}\right)
$$

et

$$
\begin{aligned}
& u_{0}+\frac{R_{\mathrm{M}}}{R_{\mathrm{g}}}-\frac{K_{\mathrm{x}}^{2}}{R_{\mathrm{g}} K_{\mathrm{R}}} \geqslant 0 \Leftrightarrow R_{\mathrm{g}}>R_{\mathrm{s}} \frac{\left(\frac{K_{\mathrm{x}}^{2}}{K_{\mathrm{R}}}-R_{\mathrm{M}}\right)}{\left(\frac{K_{\mathrm{x}}^{2}}{K_{\mathrm{R}}}+R_{\mathrm{M}}\right)} \\
& \Leftrightarrow R_{\mathrm{g}}>R_{\mathrm{s}} \\
& \text { si } R_{\mathrm{M}} \text { négligeable . }
\end{aligned}
$$

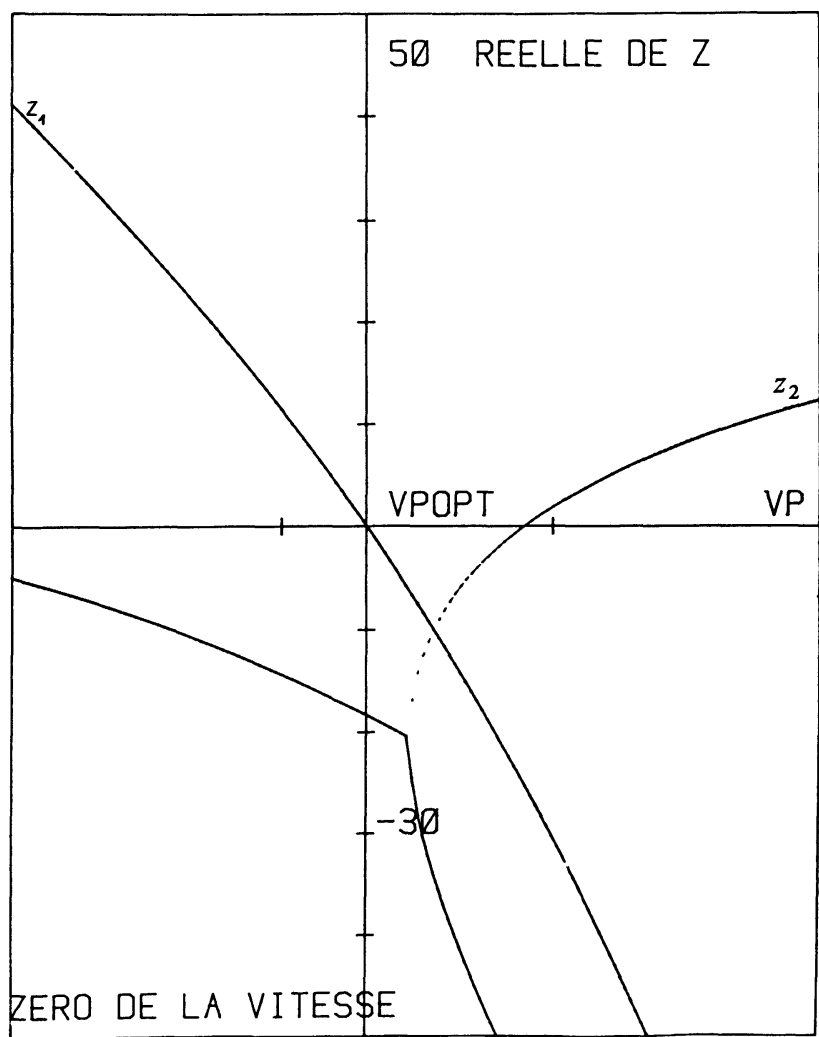

Fig. 5. - Etude des zéros de la vitesse en fonction du point de fonctionnement : $\bullet z_{1}$ (Commande par hacheur dévolteur), $\bullet z_{2}$ (Commande par hacheur survolteur).

[Study of the speed zeros in relation to the working point : - $z_{1}$ (Command by boost chopper), $\bullet z_{2}$ (Command by buck chopper).]
La première relation montre l'importance du dimensionnement relatif des composants $L$ et $C$ du convertisseur, la seconde qu'il existe une valeur limite de $\boldsymbol{R}_{\mathrm{g}}$ au-delà de laquelle les réponses ne peuvent être minimales de phase.

La figure 5 représente les variations des parties réelles des zéros $\left(z_{2}\right)$ avec le point de fonctionnement.

La figure 6 montre le déplacement des zéros lorsqu'on utilise une self de valeur moitié à celle utilisée précédemment.

Si une commande simultanée sur $u_{1}$ et $u_{2}$ est effectuée, on peut écrire du fait que $a_{0}=0$ :

$$
\begin{aligned}
\Omega(p)=u_{2} \mid b_{0} p^{2}+a_{1} \frac{u_{1}}{u_{2}} p & + \\
& +a_{2} \frac{u_{1}}{u_{2}}+b_{2} \mid \cdot \psi(p)^{-1} .
\end{aligned}
$$

Les conditions de stabilité sont alors les suivantes :

$$
\begin{gathered}
a_{1} \frac{u_{1}}{u_{2}}+b_{1}>0 \text { et } a_{2} \frac{u_{1}}{u_{2}}+b_{2}>0 \\
\frac{u_{1}}{u_{2}}>-\frac{1}{u_{0} K_{\mathrm{x}} R_{\mathrm{s}}}\left(\frac{L}{C R_{\mathrm{g}}}+R_{\mathrm{M}}-\frac{K_{\mathrm{x}}^{2}}{K_{\mathrm{R}}}\right) .
\end{gathered}
$$

Deux cas sont à envisager :

i) $\mathrm{Si} V_{\mathrm{p}}<V_{\text {popt, }}$ alors $a_{2}$ doit être négatif; ce qui conduit à :

$$
\frac{u_{1}}{u_{2}}<-\frac{b_{2}}{a_{2}} \text {. }
$$

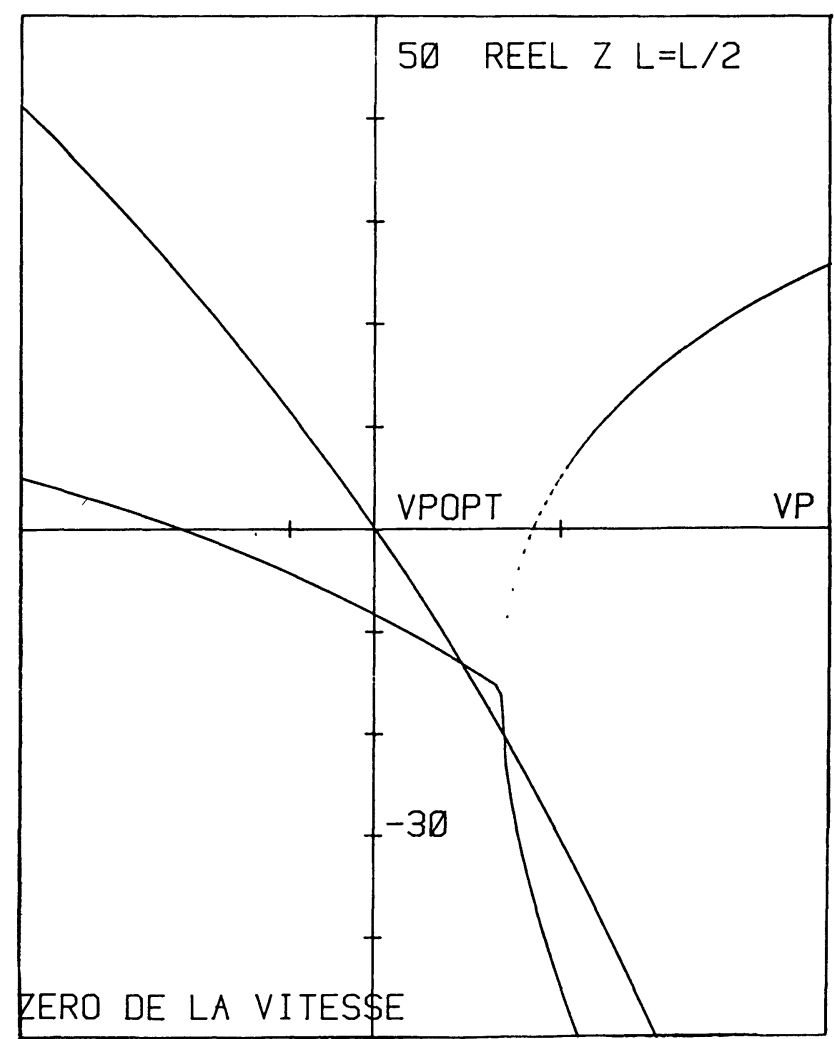

Fig. 6. - Influence de l'inductance $L$ sur les zéros.

[Sensitivity of the zeros to the $L$ value inductance.] 
ii) Sinon, $V_{\mathrm{p}}>V_{\mathrm{popt}}$ et alors $a_{2}$ doit être positif; ce qui entraîne :

$$
\frac{u_{1}}{u_{2}}>-\frac{b_{2}}{a_{2}}
$$

avec :

$$
\frac{b_{2}}{a_{2}}=-\left(U_{0}^{2} R_{\mathrm{g}}+R_{\mathrm{M}} \frac{K_{\mathrm{x}}^{2}}{K_{\mathrm{R}}}\right) \frac{1}{U_{0} K_{\mathrm{x}}\left(R_{\mathrm{s}}-R_{\mathrm{g}}\right)} .
$$

La figure 7 représente dans la partie du plan non hachurée, l'ensemble des points représentatifs du rapport de commande $\frac{U_{1}}{U_{2}}$ qui garantissent des réponses minimales de phase en fonction du point de fonctionnement $V_{\mathrm{p}}$ du générateur. Ce plan de commande définit les rapports de commandes admissibles.

La figure 8 montre l'évolution du plan de commande lorsque la valeur de la self est divisée par deux.

Généralisation de l'étude aux autres variables d'état. - L'étude des zéros peut être effectuée sur les variables d'état : courant et tension du générateur photovoltaïque.

La figure 9 définit alors le plan de commande autorisé compte tenu des contraintes inégalités obtenues sur la vitesse, la tension et le courant.

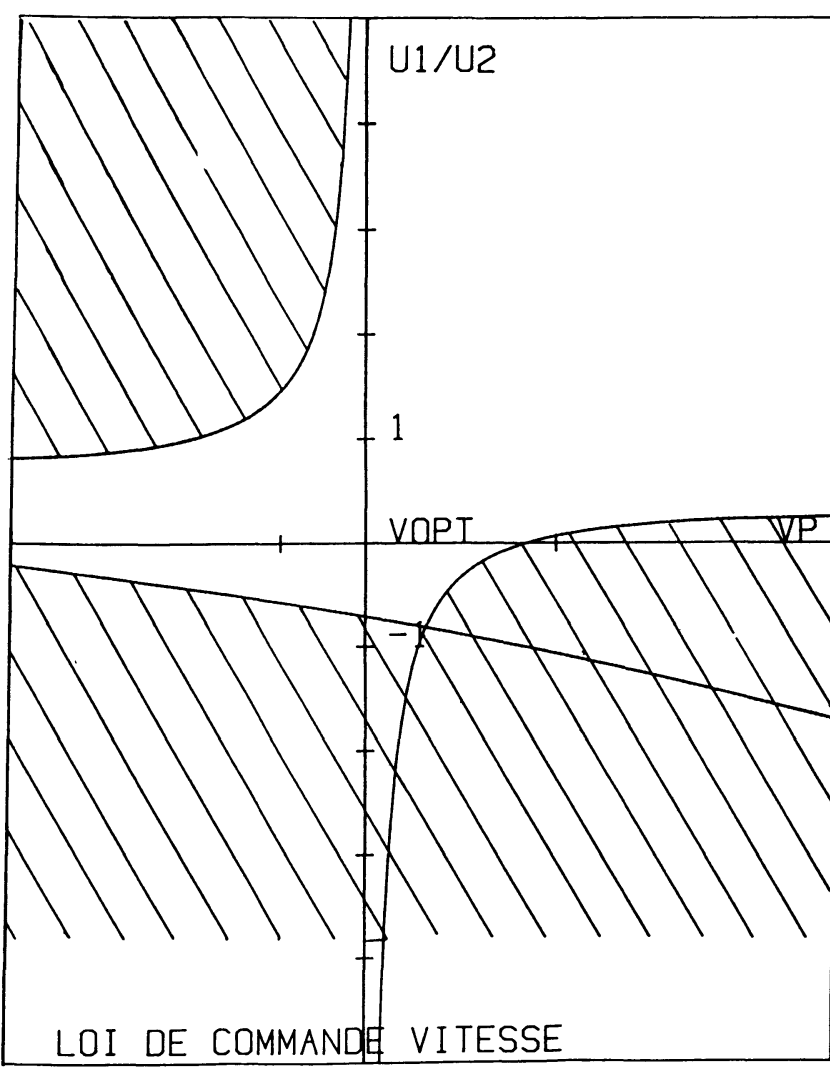

Fig. 7. - Plan de commande assurant une réponse minimale de phase de la vitesse.

[Command scheme ensuring a minimal speed phasal response.]

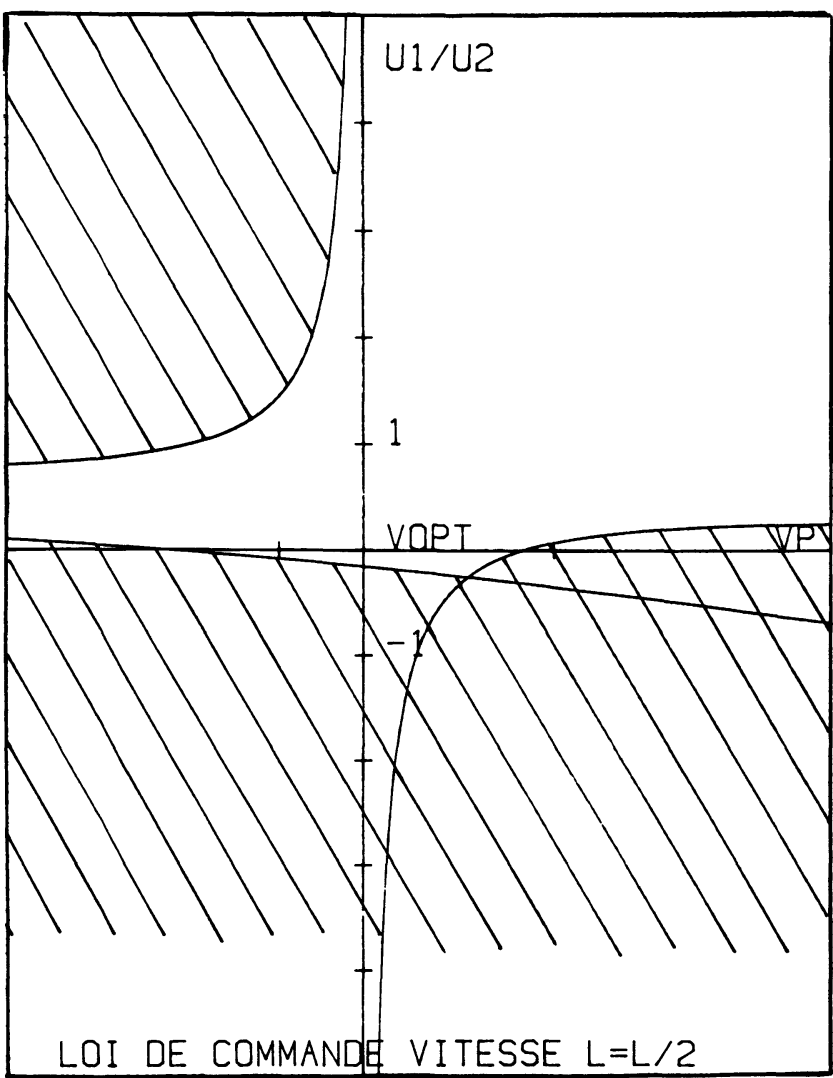

Fig. 8. - Influence de l'inductance $L$ sur le plan de commande.

[Sensitivity of the command scheme to the $L$ value inductance.]

La figure 10 montre alors la réponse obtenue en respectant le plan de commande pour $V_{\mathrm{p}}<V_{\text {opt }}$

\section{Compensation par retour d'état.}

La théorie de la représentation d'état et la compensation par retour d'état ont permis de nombreuses applications [8-10]. Elle peut être facilement appliquée aux systèmes électrotechniques [9]. On distingue deux méthodes générales permettant de calculer le compensateur d'état.

La première méthode consiste à imposer les pôles du système, l'ingénieur électronicien ayant par avance une idée des pôles optimaux compte tenu de l'expérience acquise ou des simulations effectuées. Cette méthode ne permet pas de prévoir la nature des zéros induits par ce compensateur et ne permet pas de calculer un compensateur n'agissant que sur une commande.

Nous appliquerons une seconde méthode utilisant la théorie générale de la commande optimale au sens d'un critère. Le choix d'un critère de type quadratique permet de résoudre le problème de commande optimale. Par contre, il n'existe pas de méthode générale permettant de choisir les matrices de pondération du critère. 


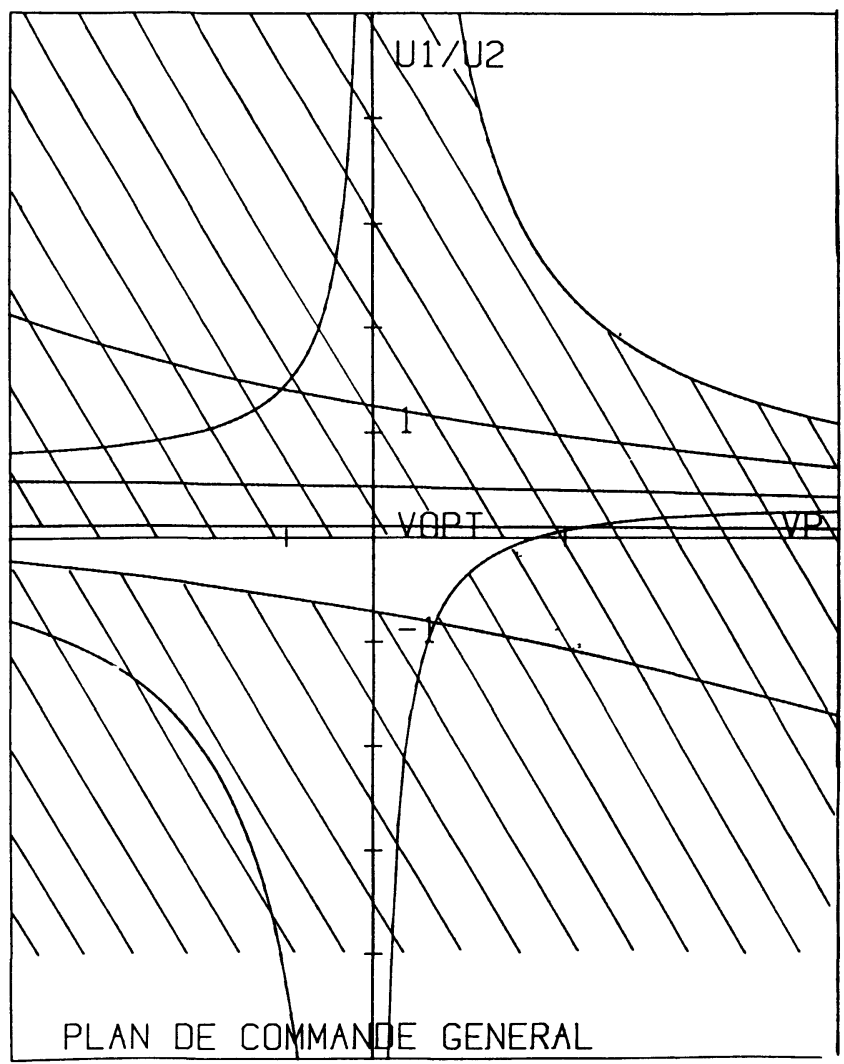

Fig. 9. - Plan de commande général.

[Sensitivity of the command scheme to the load torque.]

Le système étant multivariable, aucune théorie (à notre connaissance) ne permet de lier les zéros avec le compensateur choisi. La matrice $A$ étant modifiée par un retour d'état, il va en résulter une modification des zéros et du plan de commande que nous nous proposons d'étudier [11].

Régulateur optimal minimisant un critère quadratique. - La deuxième méthode permettant d'optimiser la réponse dynamique d'un système fait appel à la notion de critère. Nous étudierons d'une façon générale le double compensateur.

Le problème général de l'optimisation dynamique consiste à minimiser sur un horizon fini ou infini un critère $J$ fonction du vecteur d'état et de la commande :

$$
J=\int_{0}^{t} \phi|x(t), u(t)| \mathrm{d} t
$$

ou

$$
J=\int_{0}^{\infty} \phi|x(t), u(t)| \mathrm{d} t
$$

Compte tenu des contraintes inégalités différentielles :

$$
\dot{x}=A x+B u=f|x(t), u(t)| .
$$

Soit $H$ l'hamiltonien défini par :

$$
H=\phi|x(t), u(t)|+P^{T}(t) f|x(t), u(t)| .
$$

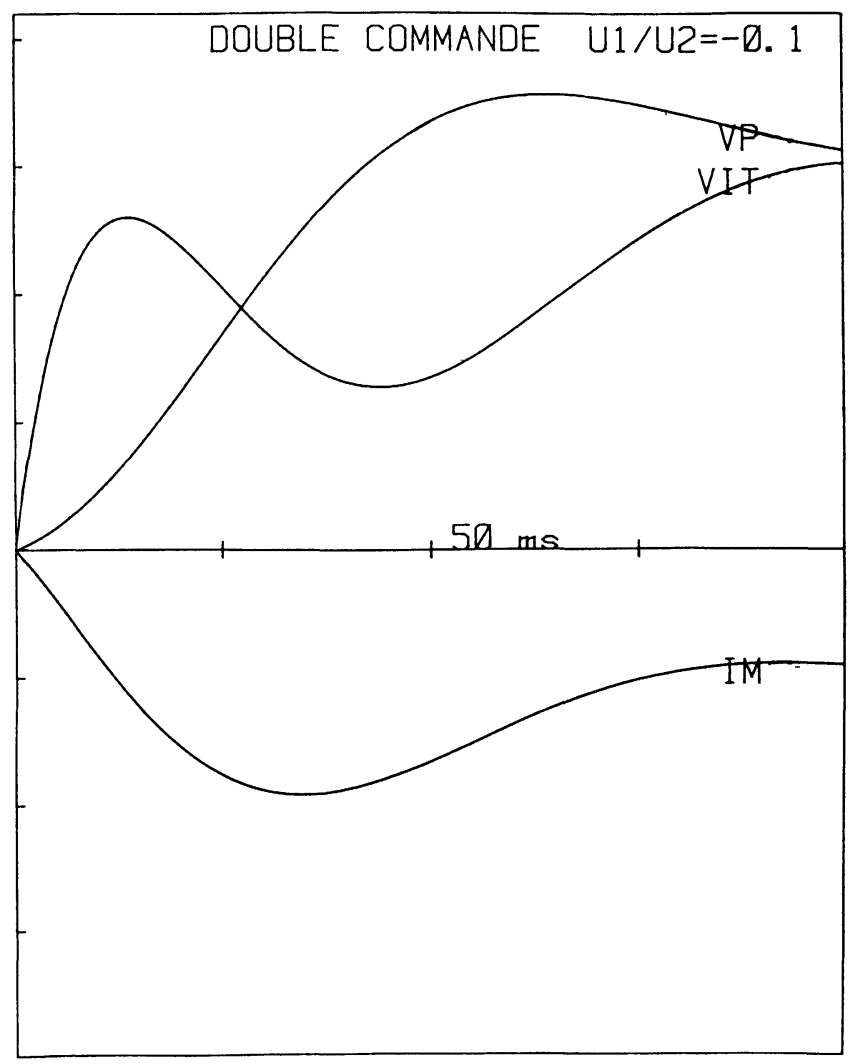

Fig. 10. - Réponse du système minimal de phase $V_{\mathrm{p}}<V_{\text {opt }}$

[General command scheme.]

Le principe du maximum de Pontriaguine nous permet de définir la commande optimale $u(t)$ telle que

$$
\frac{\partial H}{\partial u}=0 \text {. }
$$

La fonction de coût $J$ est alors minimum et le système vérifie le système d'équations de Hamilton Pontriaguine

$$
\dot{x}(t)=\frac{\partial H}{\partial P} \quad \dot{P}(t)=-\frac{\partial H}{\partial x} .
$$

Nous obtenons un système de trois équations dont la solution dépend du type de critère.

Critère quadratique. - Dans le cas où la fonction de coût est un critère quadratique

$$
J=\frac{1}{2} \int_{0}^{\infty}\left(x^{T} Q x+u^{T} R u\right) \mathrm{d} t
$$

l'hamiltonien s'écrit :

$$
H=\frac{1}{2}\left(x^{T} Q x+u^{T} R u\right)+P^{T} A x+P^{T} B u .
$$

Il est extrêmum pour :

$$
\nabla_{u} H=0=R u+B^{T} P .
$$


La commande optimale est donnée par :

$$
U_{\mathrm{opt}}=-R^{-1} B^{T} P .
$$

$\mathrm{Si}$ les relations de Hamilton Pontriaguine sont appliquées, nous obtenons :

$$
\left\{\begin{array}{l}
\dot{P}=-A^{T} P-Q x \\
\dot{x}=A x-B R^{-1} B^{T} P .
\end{array}\right.
$$

Ce qui conduit à poser :

$$
P(t)=H X(t)
$$

d'où

$$
u=K x \quad \text { et } \quad K=-R^{-1} B^{T} H .
$$

En reportant dans le système d'équations de Hamilton Pontriaguine, la matrice $H$ est la solution de l'équation de Ricatti :

$$
H A+A^{T} H-H S H+Q=0
$$

avec $S: B R^{-1} B^{T}$.

Le problème est de choisir les matrices $Q$ et $R . Q$ doit être définie non négative et $R$ définie positive.

D'une façon générale, il n'existe pas de méthode pour choisir les matrices $Q$ et $R$. Le choix des matrices $Q$ et $R$ le plus utilisé est celui de Brison.

$$
\begin{aligned}
& q_{i i}=\frac{1}{\left(\Delta x_{i \max }\right)^{2}}, q_{i j}=0 \quad i \neq j \\
& r_{i i}=\frac{1}{\left(\Delta u_{i \max }\right)^{2}}, r_{i j}=0 \quad i \neq j
\end{aligned}
$$

$\Delta x_{i \max }$ et $\Delta u_{i \max }$ sont les valeurs maximales autorisées des états et des commandes respectivement.

Résultats expérimentaux. - L'utilisation de hacheurs commandés par le rapport cyclique impose une amplitude de la commande nécessairement inférieure à 1 . Nous avons choisi une matrice $R_{0}$ diagonale tenant compte d'un compensateur sur les deux commandes

$$
R_{0}=\left|\begin{array}{rr}
10 & 0 \\
0 & 10
\end{array}\right|
$$

Le choix de $Q$ dépend des variations des variables d'état. La procédure expérimentale consiste à se fixer un échelon $\Delta V_{\mathrm{p}}$ de la variation de tension du générateur; on en déduit alors la variation $\Delta \omega$ correspondante par la relation

$$
\Delta V_{\mathrm{p}} \frac{\partial P_{\mathrm{g}}}{\partial V_{\mathrm{p}}}-\Delta \omega \frac{\partial P_{\mathrm{R}}}{\partial \omega}=0
$$

qui traduit le transfert statique de puissance $\left(\Delta P_{\mathrm{g}}=\Delta P_{\mathrm{R}}\right)$ avec :

$$
\begin{aligned}
& \frac{\partial P_{\mathrm{g}}}{\partial v_{\mathrm{p}}}=V_{\mathrm{p} 0}\left(\frac{1}{R_{\mathrm{s}}}-\frac{1}{R_{\mathrm{g}}}\right) \\
& \frac{\partial P_{\mathrm{R}}}{\partial \omega}=2 K_{\mathrm{R}} \Omega_{0} .
\end{aligned}
$$

La matrice $Q$ sera donc choisie de la forme

$$
Q=\left|\begin{array}{ccc}
Q_{11} & & 0 \\
0 & Q_{22} & Q_{33}
\end{array}\right|
$$

avec

$$
\begin{aligned}
& Q_{11}=\left(\frac{1}{\Delta V_{\mathrm{p}}}\right)^{2} \\
& Q_{33}=\left(\frac{1}{\Delta \omega}\right)^{2} .
\end{aligned}
$$

Quant au terme $Q_{22}$, il sera pris arbitrairement, sachant que plus ce terme sera grand, plus la variable d'état $i_{\mathrm{m}}$ sera contrôlée. Le choix ne pourra être fait que par simulations successives.

$Q$ et $R$ étant choisis, l'équation algébrique de Ricatti est résolue par la méthode de Barraud utilisant la fonction signe d'une matrice. L'algorithme converge en 80 itérations et la précision obtenue sur la matrice est inférieure à $10^{-2}$.

La commande $u$ est de la forme $u=e+K x$. K est alors une matrice $(m, n) m=2$ nombre de commandes et $n$ dimension du système

$$
e=\left|\begin{array}{l}
e_{1} \\
e_{2}
\end{array}\right| .
$$

Comme nous l'avons vu précédemment, les commandes $e_{1}$ et $e_{2}$ sont déterminées pour un échelon $\Delta V_{\mathrm{p}}$ donné par le système de $n+1$ équations

$$
\begin{gathered}
\Delta V_{\mathrm{p}} \frac{\partial P_{\mathrm{g}}}{\partial V_{\mathrm{p}}}-\Delta \omega \frac{\partial P_{\mathrm{R}}}{\partial \omega}=0 \\
|A+B K|\left|\begin{array}{c}
\Delta V_{\mathrm{p}} \\
\Delta i_{\mathrm{m}} \\
\Delta \omega
\end{array}\right|+B\left|\begin{array}{l}
e_{1} \\
e_{2}
\end{array}\right|=0 .
\end{gathered}
$$

Les résultats obtenus (Fig. 11) montrent la très nette amélioration de la dynamique du système.

Les pôles sont alors réels :

- 913,60

- $\quad 10,65$

$-1140$

Le rapport des commandes $e_{1} / e_{2}=-0,98$, ce qui entraîne des zéros stables pour la tension $V_{\mathrm{p}}$ et la vitesse.

Les zéros de la tension du générateur sont :

$-8235,61$

- $\quad 10,35$

le zéro de la vitesse :

- 10,43 . 


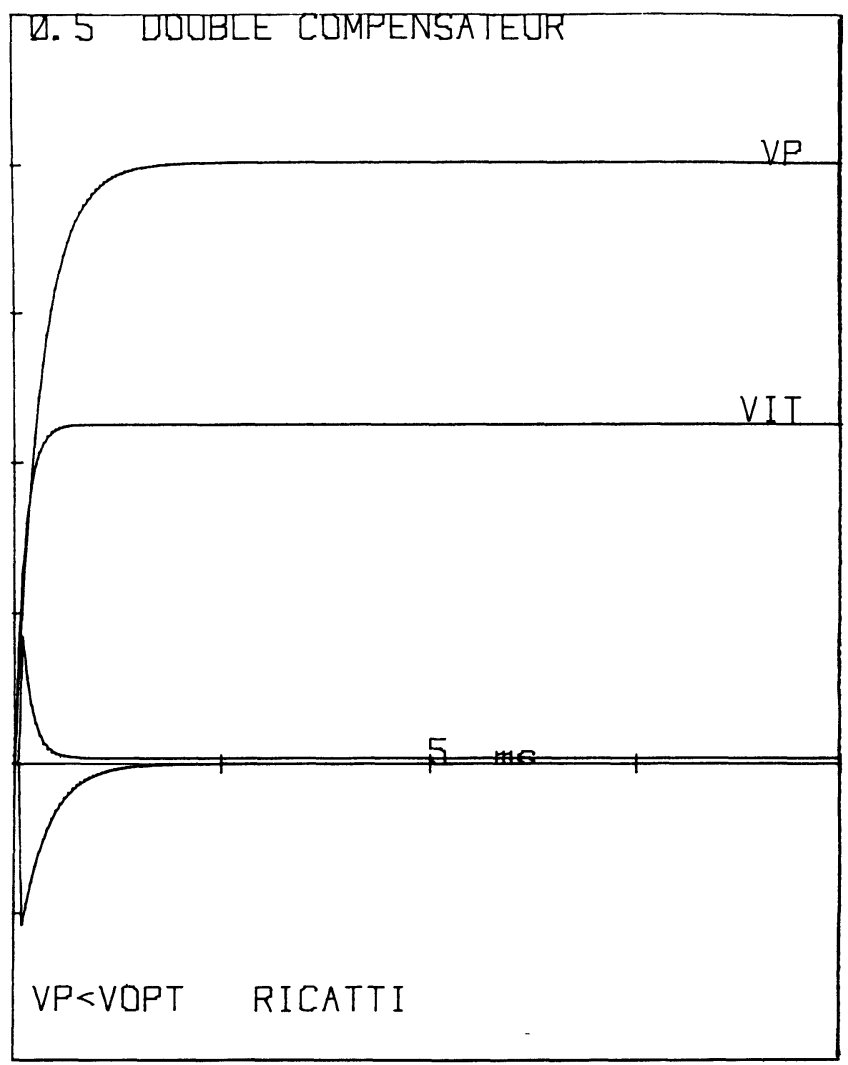

Fig. 11. - Réponse optimale.

[Optimal transient using state feedback.]

Les zéros sont stables et la réponse est toujours minimale de phase. Une étude comparée du zéro de la vitesse en fonction du rapport $u_{1} / u_{2}$ montre que si le zéro varie dans une large plage pour le système non compensé, il est pratiquement constant pour le système compensé. Cette méthode nous permet d'autre part de respecter l'amplitude maximum de la commande compte tenu de l'échelon $\Delta V_{\mathrm{p}}$ que l'on a fixé (ou $\Delta P_{\mathrm{g}}$ puisque $\Delta P_{\mathrm{g}}$ est une fonction linéaire de $\Delta V_{\mathrm{p}}$ ). L'algorithme décrit ci-contre permet de calculer le compensateur optimal compte tenu des contraintes $\Delta U_{i \max }$ et $\Delta P_{\mathbf{g}}$.

La simulation (Fig. 11) des réponses dynamiques par la méthode de Runge et Kutta montre une réponse amortie non oscillatoire dont le temps de réponse est très rapide. La comparaison avec les résultats du paragraphe précédent montre la très nette amélioration de la dynamique du système. On montre que les réponses dynamiques sont pratiquement indépendantes $d u$ point de fonctionnement si les coefficients $K_{1}, K_{2}$, $K_{3} d u$ compensateur varient en fonction de la tension $V_{\mathrm{p} 0}$ suivant les courbes indiquées (Fig. 12).

\section{Conclusion.}

Pour assurer le transfert optimal de puissance entre une source de puissance finie et un récepteur, il est
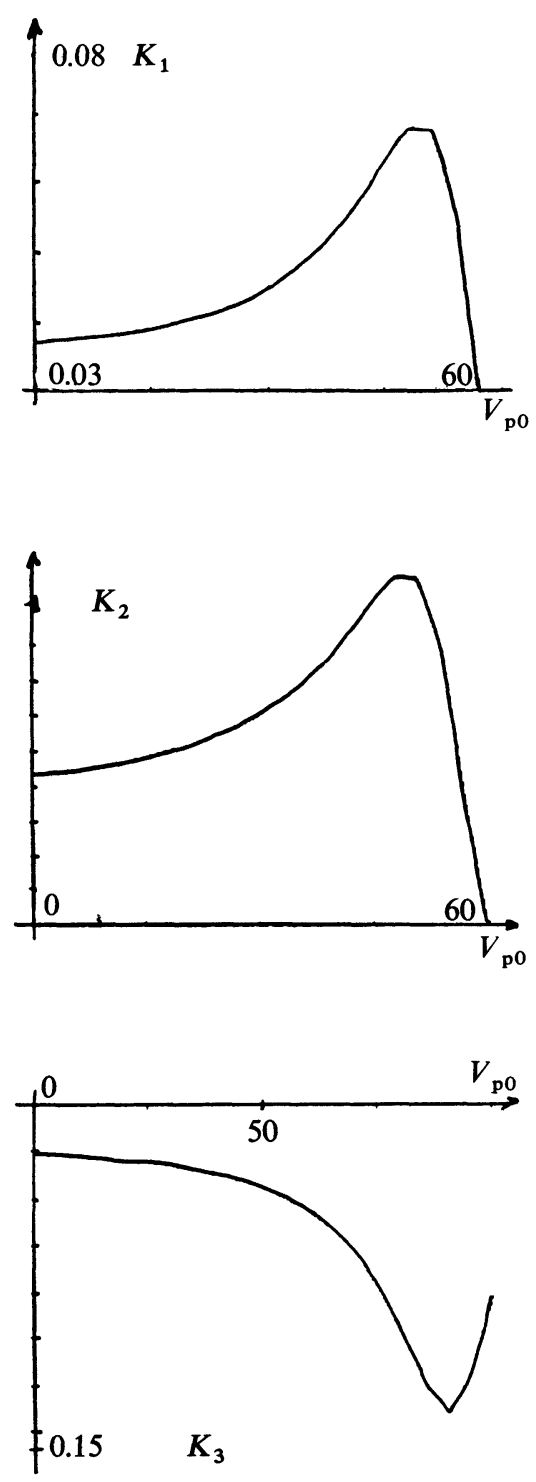

Fig. 12. - Variation des coefficients du compensateur avec la tension $V_{\mathrm{po}}$.

[Variation of the compensator coefficients with $V_{\mathrm{p} 0}$.]

nécessaire d'utiliser un convertisseur statique. L'étude de ce transfert en régime dynamique fait apparaître des réponses non minimales de phase des variables d'état dues à la présence de zéros à partie réelle positive dans la fonction de transfert. La solution proposée pour éviter ce phénomène consiste à choisir un convertisseur ayant deux degrés de liberté. Un plan de commande a été établi pour garantir des réponses minimales de phase. De plus, le rôle de l'élément de stockage tampon a été mis en évidence et sa valeur minimum déterminée.

De façon à améliorer les performances dynamiques nous avons utilisé une compensation par retour d'état. La méthode consistant à minimiser un critère quadratique assure des réponses minimales de phase dans le cas étudié. 
Cette étude qui a été menée sur un générateur photovoltaïque et un récepteur électrique est généralisable à tout générateur de puissance finie couplé à un récepteur. Il est nécessaire de faire une telle étude pour tous ces types de système afin d'éviter des réponses non minimales de phase qui engendrent des inversions de couple ou de courant susceptibles de détériorer le système.

Bibliographie

[1] Barlaud, Pradal, Requier, Recherche de la Puissance Optimale Délivrée par un Générateur Photovoltaïque à l'Aide d'un Dispositif Microprogrammé. Photovoltaic Solar Energy Conference, Cannes, 1980.

[2] Barlaud, Requier, Rouan, Optimisation of Photovoltaic Solar Pump by Means of Static Converters Driven by Microprocessor. IEEE Photovoltaïque Specialist Conference Orlando, mai 1981.

[3] Decaulne, Gille, Pelegrin, Introduction aux Systèmes Asservis Adaptatifs (DUNOD) 1976.

[4] Middle Brook, R.D. and Slobodan CuK, A General Unified Approach to Modelling Switching Converter Power Stages - INTJ Electronics, 1977, Vol. 42 $\mathrm{n}^{\mathrm{o}} 6$.

[5] Mac Farlane, J. and Karcanias, N., Engineering Department, University of Cambridge Relationships between State-space and Frequency, Reponse Concepts, Multivariable Control Systems : General Aspects.
[6] Commault, C., Quelques Aspects Structurels de la Commande des Systèmes Multivariables. Thèse d'Etat Sciences INPG Grenoble, Février 1983.

[7] Davison, E.J., Some Properties of Minimum Phase Systems and "Squared down" Systems IEEE Trans. Automatic Control AC-28 no 2 (1983).

[8] Fossard, Commande des Systèmes Multidimensionnels, (Dunod) 1972.

[9] De Fornel, B., Pietrzak David, M., Gauvrit, M., State Space Compensation Applied to the Control of a Current-fed Induction Motor, IEE Proc., Vol. $129 \mathrm{n}^{\circ} 4$ (1982).

[10] ZAKI, A., Utilisation de la Commande Optimale pour la Détermination d'un Pilote Automatique d'Avion. Thèse de Docteur Ingénieur. Ecole Supérieure d'Aéronautique et de l'Espace. Toulouse, 1980.

[11] Barlaud, De Fornel, GAUVRIT, ReQuier, Etude Dynamique du Transfert Optimum de Puissance entre un Générateur de Puissance Finie et un Récepteur. IMACS, Nantes, 1983. 